\title{
Generating and detecting topological phases with higher Chern number
}

\author{
Abhijeet Alase* \\ Institute for Quantum Science and Technology, and Department of Physics and Astronomy, \\ University of Calgary, Alberta T2N 1N4, Canada \\ David L. Feder \\ Institute for Quantum Science and Technology, and Department of Physics and Astronomy, \\ University of Calgary, Calgary, Alberta, Canada T2N $1 N_{4}$
}

\begin{abstract}
Topological phases with broken time-reversal symmetry and Chern number $|\mathcal{C}| \geq 2$ are of fundamental interest, but it remains unclear how to engineer the desired topological Hamiltonian within the paradigm of spin-orbit-coupled particles hopping only between nearest neighbours of a static lattice. We show that phases with higher Chern number arise when the spin-orbit coupling satisfies a combination of spin and spatial rotation symmetries. We leverage this result both to construct minimal two-band tight-binding Hamiltonians that exhibit $|\mathcal{C}|=2,3$ phases, and to show that the Chern number of one of the energy bands can be inferred from the particle spin polarization at the high-symmetry crystal momenta in the Brillouin zone. Using these insights, we provide a detailed experimental scheme for the specific realization of a time-reversal-breaking topological phase with $|\mathcal{C}|=2$ for ultracold atomic gases on a triangular lattice subject to spin-orbit coupling. The Chern number can be directly measured using Zeeman spectroscopy; for fermions the spin amplitudes can be measured directly via time of flight, while for bosons this is preceded by a short Bloch oscillation. Our results provide a pathway to the realization and detection of novel topological phases with higher Chern numbers in ultracold atomic gases.
\end{abstract}

\section{INTRODUCTION}

Since the breakthrough provided by the quantum Hall effect, the systems that display topological features in the absence of an external magnetic field have been at the center stage in research in condensed matter physics. The landmark discovery of topological insulators as well as quantum anomalous Hall systems established spin-orbit (SO) coupling as the dominant mechanism behind topological band inversion [1]. In contrast to time-reversal invariant topological systems including two-dimensional (2D) topological insulators, protected phases characterized by higher Chern numbers $|\mathcal{C}| \geq 2$ are theoretically possible in time-reversal broken systems [2]. (Note that in this work the Chern number is understood to mean the first Chern number.) Such systems are predicted to show rich physics [3-6] and in the solid state have potential applications such as in low-power consumption electronics [7].

Experimentally, only a handful of solid-state materials have been synthesized that exhibit $|\mathcal{C}| \geq 2$ phases [8-11], though photonic platforms have also met with some success [12]. One route to the realization of higher Chern number phases in the solid state is by the use of layered systems [11, 13, 14], such as thin films of topological insulators [15-17], in which the Chern numbers of individual energy bands add up to yield an overall higher Chern number. Much of the theoretical research on higher Chern number bands has focused on model tight-binding Hamiltonians that host such phases [14, 18-

\footnotetext{
* Corresponding author: abhijeet.alase1@ucalgary.ca
}

26]. Most of these Hamiltonians either require high angular momentum bonding, next-nearest-neighbor (nnn) terms, or complicated geometries that are difficult to realize experimentally. For instance, the smallest known nearest-neighbor $(\mathrm{nn})$ Hamiltonian that displays a $\mathcal{C}=2$ phase requires $d$ orbitals [27] or six bands [18]. Identification of solid-state material candidates with $|\mathcal{C}| \geq 2$ has generally relied on $a b$ initio calculations [8, 17, 27, 28]. Despite their importance, the mechanisms that lead to higher Chern number bands in realistic systems remain poorly understood.

Ultracold atoms trapped in optical lattices hold the promise to realize novel and fascinating topological phases [29-55]. In contrast to solid-state systems, a diverse set of experimental techniques are available for controlling and tuning the Hamiltonians governing ultracold atomic gases. For example, it is straightforward to generate a wide variety of regular and non-Bravais lattices [56-58], including those with high or multiple orbital bands $[59,60]$. Recent experiments $[46,54,55]$ have shown that it is possible to realize SO coupling for ultracold atoms trapped in an optical lattice by using a Raman coherence technique [61], but marrying the periodicity of the SO interactions to that of the underyling optical lattice is not straightforward for all geometries. To date, only $\mathcal{C}= \pm 1$ topologically non-trivial phases have been realized experimentally in these systems.

The estimation of the Chern number in ultracold atom environments also remains a challenge, as routine transverse conductance measurements on solid-state systems are not straightforward to implement in ultracold atomic gases [35, 41, 45, 47, 49]. Whereas several approaches have been proposed [29-45] and implemented [47-55] for the detection of topological order in ultracold atoms, 
most of them either do not apply to SO coupled systems or rely on experimentally challenging procedures such as mapping the Berry curvature over the entire Brillouin zone (BZ). Furthermore, distinguishing a topological phase with Chern number $\mathcal{C}= \pm 2$ from a trivial one with $\mathcal{C}=0$ can be challenging using approaches that are easier to implement experimentally [35, 45]. The difficulty stems in part from the fact that weakly interacting bosons condense to a single point in the BZ at zero temperature, rather than occupying a full energy band like their fermionic counterparts, necessitating a dynamical approach to the detection of the topological order. Moreover, it appears that topological phases with Chern number $\mathcal{C}= \pm 2$ cannot be realized using ultracold atoms on a square lattice; for example, a proposed timereversal-breaking two-band model [19] requires strong next-nearest neighbor hopping.

In this work, we present two main results. First, we identify a simple mechanism that can give rise to higher values of the Chern number $|\mathcal{C}| \geq 2$ in individual bands, for time-reversal breaking systems. Second, we leverage the first result to propose an experimental scheme using ultracold atomic gases to realize a nearest-neighbor Hamiltonian that exhibits a topological phase with Chern number $|\mathcal{C}|=2$.

The first result is the derivation of a relation between the Chern number $(\bmod 2 n, n \in \mathbb{Z})$ and the spin polarization at the high-symmetry points in the BZ of a SO coupled Hamiltonian supported on a Bravais lattice with $2 n$-fold rotation symmetry $C_{2 n}$. The key insight is a relation between the Chern number and the Berry-Zak phase of a closed loop that connects high-symmetry points of the BZ, Eq. (15). The relation can be extended to systems with any number of internal states, and to other non-Bravais lattices (i.e. Bravais lattices with attached basis) in a straightforward way.

The second result leverages the Chern-spin polarization relation to construct, among others, a minimal twoband nn tight-binding Hamiltonian on a triangular lattice that exhibits topological phases with $\mathcal{C}= \pm 2$, Eq. (20). We provide a detailed proposal for realizing this model with ultracold atoms in optical lattices, and for the measurement of the lower-band Chern number. Our scheme judiciously combines the existing techniques for the generation of a triangular optical lattice [62] with an extension of the SO coupling scheme implemented on a square lattice using Raman coherence [54]. We design a novel scheme for the detection of the Chern number in bosonic systems using Bloch oscillations and Zeeman spectroscopy. Both these techniques are experimentally well established and widely employed [63-65]. For fermionic systems, the Chern number can be determined directly by time-of-flight (TOF) imaging [66], without the need of Bloch oscillations.

Whereas the Hamiltonian that we construct and propose to realize satisfies a particular spin-space rotation symmetry, the resulting topological phase is protected against all particle number preserving perturbations re- gardless of whether they obey the aforementioned symmetry. This is a straightforward consequence of the fact that the topological phase is characterized by the Chern number. In this sense, the Hamiltonian we construct belongs to the class of Chern insulators, and not crystalline topological insulators.

The paper is organized as follows. Section II discusses a symmetry principle that can yield topological two-band Hamiltonians with higher Chern numbers for a given band. A key relation that links the spin polarization at high-symmetry points in the BZ is derived in Sec. II A, and several examples of tight-binding Hamiltonians with $|\mathcal{C}| \geq 2$ are given in Sec. II B. Section III is devoted to a detailed proposal for the implementation (Sec. III A), its analysis (Secs. III B and III C) and a detection scheme (Sec. III D) for a topological Hamiltonian on a triangular lattice with band Chern number $|\mathcal{C}|=2$. The results are summarized in Sec. IV.

\section{SYMMETRY PRINCIPLE YIELDING HIGHER CHERN NUMBER}

\section{A. Chern-spin polarization relation}

In this section, we derive an important relationship between the Chern number and the spin polarization at high-symmetry points in the BZ. Assume that the system is described by a gapped $2 \times 2$ tight-binding Bloch Hamiltonian $H(\mathbf{k})$, with symmetry property

$$
H(S \mathbf{k})=U H(\mathbf{k}) U^{\dagger}, \quad U:=e^{-i \pi m \sigma_{z} / 2 n},
$$

where $\sigma_{z}$ is the Pauli- $z$ matrix, and $m \in\{1,2,3\}$. The symmetry group is generated by counter-clockwise rotations around the $z$ axis by angle $\pi / n$; we denote the rotation in real space by $R:=R_{z}(\pi / n)$ and in reciprocal space by $S:=R_{z}(\pi / n)$. The symmetry condition for $m=1$ is satisfied by Rashba SO coupling, which for small $|\mathbf{k}|$ has the form $\mathbf{k} \times \vec{\sigma} \cdot \hat{z}$. The symmetry for $m=2$ $(m=3)$ requires that the spin wave function of any one of the bands on the Bloch sphere rotates twice (thrice) as rapidly as the momentum vectors in the $x y$ plane of the BZ.

Let $|u(\mathbf{k})\rangle$ denote the lower band wavefunction of the Hamiltonian $H(\mathbf{k})$. Let $\overrightarrow{\mathcal{A}}(\mathbf{k})=\left\langle u(\mathbf{k})\left|\vec{\nabla}_{\mathbf{k}}\right| u(\mathbf{k})\right\rangle$ and $\mathcal{B}(\mathbf{k})=\vec{\nabla}_{\mathbf{k}} \times \overrightarrow{\mathcal{A}} \cdot \hat{z}$ denote the Berry connection and the Berry curvature, respectively of the lower energy band. The Chern number

$$
\mathcal{C}=\frac{1}{2 \pi i} \int_{\mathrm{BZ}} \mathcal{B}(\mathbf{k}) d^{2} \mathbf{k}
$$

can be related to the spin wavefunctions at the highsymmetry momenta $\Gamma$ and $M$ in two steps using an approach similar to that discussed in Ref. [67]. For any integer $\ell$, Eq. (1) dictates that the wavefunctions at crystal momenta $S^{\ell} \mathbf{k}$ and $\mathbf{k}$ satisfy $\left|u\left(S^{\ell} \mathbf{k}\right)\right\rangle=e^{i \theta_{\ell}(\mathbf{k})} U^{\ell}|u(\mathbf{k})\rangle$ 
for some real function $\theta_{\ell}$ on the BZ. Then

$$
\begin{aligned}
\mathcal{A}_{i}\left(S^{\ell} \mathbf{k}_{0}\right) & =\left.\left\langle u(\mathbf{q})\left|\partial_{q_{i}}\right| u(\mathbf{q})\right\rangle\right|_{\mathbf{q}=S^{\ell} \mathbf{k}_{0}} \\
& =\left.\left\langle u\left(S^{\ell} \mathbf{k}\right)\left|\sum_{j} S_{j i}^{-\ell} \partial_{k_{j}}\right| u\left(S^{\ell} \mathbf{k}\right)\right\rangle\right|_{\mathbf{k}=\mathbf{k}_{0}} \\
& =\left\langle u(\mathbf{k})\left|\left(U^{\dagger}\right)^{\ell} e^{-i \theta_{\ell}(\mathbf{k})} \sum_{j} S_{j i}^{-\ell} \partial_{k_{j}} e^{i \theta_{\ell}(\mathbf{k})} U^{\ell}\right| u(\mathbf{k})\right\rangle \\
& =\sum_{j} S_{i j}^{\ell}\left\langle u(\mathbf{k})\left|\left(U^{\dagger}\right)^{\ell} e^{-i \theta_{\ell}(\mathbf{k})} \partial_{k_{j}} e^{i \theta_{\ell}(\mathbf{k})} U^{\ell}\right| u(\mathbf{k})\right\rangle \\
& =\sum_{j} S_{i j}^{\ell}\left(\mathcal{A}_{j}\left(\mathbf{k}_{0}\right)+i \partial_{k_{j}} \theta_{\ell}\left(\mathbf{k}_{0}\right)\right),
\end{aligned}
$$

where $\partial_{k_{i}} \equiv \partial / \partial k_{i}$ is defined for brevity. Therefore,

$$
\overrightarrow{\mathcal{A}}\left(S^{\ell} \mathbf{k}\right)=S^{\ell}\left(\overrightarrow{\mathcal{A}}(\mathbf{k})+i \vec{\nabla}_{\mathbf{k}} \theta_{\ell}(\mathbf{k})\right) .
$$

Because the unitary operator $U$ is independent of $\mathbf{k}$, the gauge independence of the Berry curvature $\mathcal{B}(\mathbf{k})$ leads to

$$
\begin{aligned}
\mathcal{B}\left(S^{\ell} \mathbf{k}\right) & =\vec{\nabla}_{\mathbf{k}} \times \overrightarrow{\mathcal{A}}\left(S^{\ell} \mathbf{k}\right) \cdot \hat{z} \\
& =\vec{\nabla}_{\mathbf{k}} \times S^{\ell}\left(\overrightarrow{\mathcal{A}}(\mathbf{k})+i \vec{\nabla}_{\mathbf{k}} \theta_{\ell}(\mathbf{k})\right) \cdot \hat{z} \\
& =\vec{\nabla}_{\mathbf{k}} \times\left(\overrightarrow{\mathcal{A}}(\mathbf{k})+i \vec{\nabla}_{\mathbf{k}} \theta_{\ell}(\mathbf{k})\right) \cdot \hat{z} \\
& =\vec{\nabla}_{\mathbf{k}} \times \overrightarrow{\mathcal{A}}(\mathbf{k}) \cdot \hat{z}=\mathcal{B}(\mathbf{k}),
\end{aligned}
$$

where we have made use of the facts that $S^{\ell}$ is an orthogonal transformation, and that the curl of a gradient is zero. The result is $\mathcal{B}(S \mathbf{k})=\mathcal{B}(\mathbf{k})$, where $\hat{z}$ denotes the unit vector in the $z$ direction. The loop encloses an area $A(\gamma)$, which covers exactly $1 / 2 n$ of the total BZ and satisfies $\sum_{j=1}^{2 n} S^{j} A(\gamma)=\mathrm{BZ}$. By invoking Stokes' theorem, one obtains

$$
\frac{\mathcal{C}}{2 n}=\frac{1}{2 \pi i} \int_{A(\gamma)} \mathcal{B}(\mathbf{k}) d^{2} \mathbf{k}=\frac{1}{2 \pi i} \int_{\gamma} \overrightarrow{\mathcal{A}}(\mathbf{k}) \cdot d \mathbf{k}(\bmod 1),
$$

where $(\bmod 1)$ accounts for the definition of the BerryZak phase acquired over a closed loop modulo $2 \pi$.

In the next step, the Berry phase acquired over the loop $\gamma$ is related to the spin wavefunction at the points $\Gamma, M$, and $X$, as shown in Fig. 1 . The loop $\gamma$ may be decomposed into four parts,

$$
\begin{aligned}
\gamma=\left(X \rightarrow \Gamma \rightarrow X^{\prime}\right) & \cup\left(X^{\prime} \rightarrow M^{\prime}\right) \\
\cup & \left(M^{\prime} \rightarrow \Gamma \rightarrow M\right) \cup(M \rightarrow X) .
\end{aligned}
$$

The integral of the Berry connection over the second and the fourth segments on the right-hand side of Eq. (7) cancel each other, as the two segments correspond to the same paths in the BZ traversed in opposing directions. It is therefore only necessary to calculate the phases acquired over $X \rightarrow \Gamma \rightarrow X^{\prime}$ and $M^{\prime} \rightarrow \Gamma \rightarrow M$.

Recall that on the segment $M^{\prime} \rightarrow \Gamma \rightarrow M$, the wave functions satisfy $\left|u\left(S^{n-1} \mathbf{k}\right)\right\rangle=e^{i \theta(\mathbf{k})} U^{n-1}|u(\mathbf{k})\rangle$, where $\theta(\mathbf{k}):=\theta_{n-1}(\mathbf{k})$ is used for brevity. Because $M$ and $M^{\prime}$

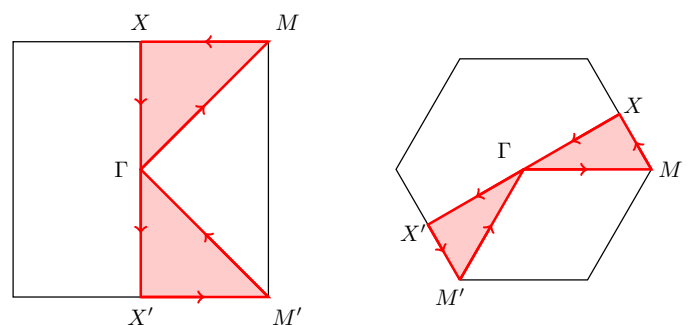

FIG. 1. (Color online) Loop $\gamma$ in the BZ of a two-dimensional Bravais lattice with $C_{4}$ (left) and $C_{6}$ (right) symmetry. The area $A(\gamma)$ enclosed by $\gamma$ is shaded in each case.

represent the same points in the BZ, $|u(M)\rangle \propto\left|u\left(M^{\prime}\right)\right\rangle$, so that

$$
\theta(\mathbf{K})=-\operatorname{Arg}\left\langle u(\mathbf{K})\left|U^{n-1}\right| u(\mathbf{K})\right\rangle, \quad \mathbf{K} \in\left\{\Gamma, M^{\prime}\right\} .
$$

The Berry phase along the path $M^{\prime} \rightarrow \Gamma \rightarrow M$ may be expressed as

$$
\int_{M^{\prime} \rightarrow \Gamma \rightarrow M} \overrightarrow{\mathcal{A}}(\mathbf{k}) \cdot d \mathbf{k}=\int_{\Gamma \rightarrow M} \overrightarrow{\mathcal{A}}(\mathbf{k}) \cdot d \mathbf{k}-\int_{\Gamma \rightarrow M^{\prime}} \overrightarrow{\mathcal{A}}(\mathbf{k}) \cdot d \mathbf{k} .
$$

Using the symmetry properties, the first term on the right hand side of Eq. (9) can be expressed as

$$
\begin{aligned}
\int_{\Gamma \rightarrow M} & \overrightarrow{\mathcal{A}}(\mathbf{q}) \cdot d \mathbf{q}=\int_{\Gamma \rightarrow M^{\prime}} \overrightarrow{\mathcal{A}}\left(S^{n-1} \mathbf{k}\right) \cdot S^{n-1} d \mathbf{k} \\
= & \int_{\Gamma \rightarrow M^{\prime}} S^{n-1}\left(\overrightarrow{\mathcal{A}}(\mathbf{k})+i \vec{\nabla}_{\mathbf{k}} \theta(\mathbf{k})\right) \cdot S^{n-1} d \mathbf{k} \\
= & \int_{\Gamma \rightarrow M^{\prime}}\left(\overrightarrow{\mathcal{A}}(\mathbf{k})+i \vec{\nabla}_{\mathbf{k}} \theta(\mathbf{k})\right) \cdot d \mathbf{k} \\
= & \int_{\Gamma \rightarrow M^{\prime}} \overrightarrow{\mathcal{A}}(\mathbf{k}) \cdot d \mathbf{k}+i\left(\theta\left(M^{\prime}\right)-\theta(\Gamma)\right),
\end{aligned}
$$

where we used Eq. (4) for $\ell=n-1$ in the second step. On substituting Eq. (10) into Eq. (9), one obtains

$$
\int_{M^{\prime} \rightarrow \Gamma \rightarrow M} \overrightarrow{\mathcal{A}}(\mathbf{k}) \cdot d \mathbf{k}=i\left(\theta\left(M^{\prime}\right)-\theta(\Gamma)\right) .
$$

The symmetry of the points $S^{n-1} \Gamma=\Gamma$ and $S^{n-1} M^{\prime}=$ $M$ enforces the condition that $|u(\Gamma)\rangle$ and $|u(M)\rangle$ are eigenstates of the generator of $U$, namely $\sigma_{z}$. We can use this fact to simplify Eq. (11) to

$$
\int_{M^{\prime} \rightarrow \Gamma \rightarrow M} \overrightarrow{\mathcal{A}}(\mathbf{k}) \cdot d \mathbf{k}=\frac{i \pi m(n-1)}{2 n}\left(\left\langle\sigma_{z}\right\rangle_{M}-\left\langle\sigma_{z}\right\rangle_{\Gamma}\right),
$$

where $\left\langle\sigma_{z}\right\rangle_{\mathbf{K}}=\left\langle u(\mathbf{K})\left|\sigma_{z}\right| u(\mathbf{K})\right\rangle$ is the spin polarization. The same calculation can be repeated for the path $X \rightarrow$ $\Gamma \rightarrow X^{\prime}$, which yields

$$
\int_{X \rightarrow \Gamma \rightarrow X^{\prime}} \overrightarrow{\mathcal{A}}(\mathbf{k}) \cdot d \mathbf{k}=\frac{i \pi m}{2}\left(\left\langle\sigma_{z}\right\rangle_{\Gamma}-\left\langle\sigma_{z}\right\rangle_{X}\right) .
$$

Adding all contributions and substituting in Eq. (6) yields

$$
\frac{\mathcal{C}}{2 n}=\frac{m}{4 n}\left[\left\langle\sigma_{z}\right\rangle_{\Gamma}+(n-1)\left\langle\sigma_{z}\right\rangle_{M}-n\left\langle\sigma_{z}\right\rangle_{X}\right] \quad(\bmod 1) .
$$


Finally we multiply the whole equation by $2 n$ to obtain the "Chern-spin polarization relation"

$$
\mathcal{C}=\frac{m}{2}\left[\left\langle\sigma_{z}\right\rangle_{\Gamma}+(n-1)\left\langle\sigma_{z}\right\rangle_{M}-n\left\langle\sigma_{z}\right\rangle_{X}\right] \quad(\bmod 2 n) .
$$

This is the first main result of the present work.

The Chern-spin polarization relation (15) provides insight into the mechanisms responsible for the emergence of energy bands characterized by $|\mathcal{C}|=2,3$. As each $\left\langle\sigma_{z}\right\rangle_{\mathbf{K}}$ take values in $\{1,-1\}$, Eq. (15) implies that $\mathcal{C}$ must be an even integer for $m=2$ and any value of $n$. Therefore, a Hamiltonian satisfying the $m=2$ symmetry after undergoing a topological band inversion typically enters a $|\mathcal{C}|=2$ phase. Similarly, for a triangular lattice Hamiltonian obeying the $m=3$ symmetry, a topological band inversion typically leads to a $|\mathcal{C}|=3$ phase. Note that a high value of the Chern number, i.e. $|\mathcal{C}|=2,3$ is not guaranteed, but possible, for Hamiltonians obeying the $m=1$ symmetry after undergoing topological band inversion. These observations form a basis for our construction of higher Chern number Hamiltonians in the next section.

\section{B. Engineering topological Hamiltonians}

The Chern-spin polarization relation is a powerful tool for engineering topological Hamiltonians, as we demonstrate next by constructing a tight-binding Hamiltonian with a $|\mathcal{C}|=2$ band. Let $\mathbf{a}_{1}$ and $\mathbf{a}_{2}$ be the lattice vectors, with angular separation $2 \pi / 3$, of a triangular Bravais lattice, and $\mathbf{a}_{3}:=-\mathbf{a}_{1}-\mathbf{a}_{2}$. The expression for a general two-band SO coupled Hamiltonian is

$$
H(\mathbf{k})=h_{x} \sigma_{x}+h_{y} \sigma_{y}+h_{z} \sigma_{z}
$$

where $h_{x}, h_{y}$, and $h_{z}$ are $\mathbf{k}$-dependent coefficients, and a spin-independent term proportional to the identity is omitted as it has no effect on the Chern number. Our strategy is to construct a gapped Hamiltonian which obeys the $m=2$ symmetry and satisfies $\operatorname{sgn}\left(h_{z}(M)\right)=$ $-\operatorname{sgn}\left(h_{z}(\Gamma)\right)$. By Eq. (15), such a Hamiltonian must have $|\mathcal{C}|=2$. To restrict the Hamiltonian to nn terms, each of the coefficients $h_{x}, h_{y}$ and $h_{z}$ must be linear combinations of $\left\{\cos k_{q}, \sin k_{q}, q=1,2,3\right\}$, with $k_{q}=\mathbf{k} \cdot \mathbf{a}_{q}$. As $\sigma_{z}$ satisfies $U \sigma_{z} U^{\dagger}=\sigma_{z}$, the constraint on $h_{z}$ is $h_{z}(\mathbf{k})=h_{z}(S \mathbf{k})$, which restricts the possible values to

$$
h_{z}=M_{z}+2 t_{z} \sum_{q} \cos \left(k_{q}\right), \quad M_{z}, t_{z} \in \mathbb{R} .
$$

Note that a term proportional to $\sum_{q} \sin \left(k_{q}\right)$ in $h_{z}$ breaks the required symmetry of the Hamiltonian. A simple check is to note that we require $h_{z}\left(S^{3} \mathbf{k}\right)=h_{z}(-\mathbf{k})=$ $h_{z}(\mathbf{k})$, and the latter equality is not obeyed by a term proportional to $\sum_{q} \sin \left(k_{q}\right)$ in $h_{z}$. The constraints on the coefficients of $\sigma_{x}$ and $\sigma_{y}$ imposed by the symmetry lead to

$$
\begin{aligned}
& h_{x}=2 t_{\mathrm{so}} \sum_{q} \cos \left(k_{q}\right) \cos (2 q \pi / 3), \\
& h_{y}=-2 t_{\mathrm{so}} \sum_{q} \cos \left(k_{q}\right) \sin (2 q \pi / 3), \quad t_{\mathrm{so}} \in \mathbb{R},
\end{aligned}
$$
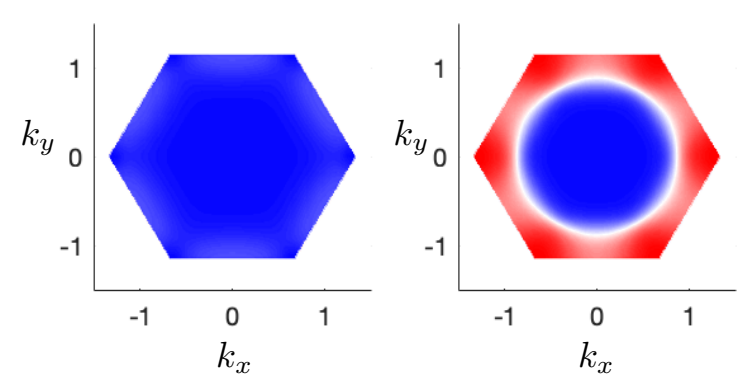

FIG. 2. (Color online) Color map of spin polarization over the BZ for the trivial (left) and the topological (right) phase with $\mathcal{C}=-2$. The crystal momenta $k_{x}$ and $k_{y}$ are in units of $\pi / a$, with $a=\left|\mathbf{a}_{q}\right|$ denoting the lattice constant. The bright red (blue) color represents $+1(-1)$ value of the spin polarization $\left\langle\sigma_{z}\right\rangle_{\mathrm{k}}$. Parameters used were $t_{\mathrm{so}}=0.5, t_{z}=1$, $M_{z}=4$ (trivial phase) and $M_{z}=1$ (topological phase).

which completes the construction of the Hamiltonian. We note that for small values of $|\mathbf{k}|$, this exotic SO coupling term

$$
\begin{aligned}
h_{x} \sigma_{x}+h_{y} \sigma_{y} & \propto\left(k_{x}^{2}-k_{y}^{2}-2 \sqrt{3} k_{x} k_{y}\right) \sigma_{x} \\
& +\left(\sqrt{3} k_{x}^{2}-\sqrt{3} k_{y}^{2}+2 k_{x} k_{y}\right) \sigma_{y},
\end{aligned}
$$

is quadratic in $k_{x}, k_{y}$. After simplification using trigonometric identities, the terms reduce to

$$
\begin{aligned}
& h_{x}=t_{\mathrm{so}}\left(2 \cos \left(k_{3}\right)-\cos \left(k_{1}\right)-\cos \left(k_{2}\right)\right), \\
& h_{y}=-\sqrt{3} t_{\mathrm{so}}\left(\cos \left(k_{1}\right)-\cos \left(k_{2}\right)\right), \\
& h_{z}=M_{z}+2 t_{z}\left(\cos \left(k_{1}\right)+\cos \left(k_{2}\right)+\cos \left(k_{3}\right)\right) .
\end{aligned}
$$

For $t_{\mathrm{so}} \neq 0$ and $t_{z}>0$, this Hamiltonian is gapped except for $M_{z} / t_{z} \in\{-6,2,3\}$. Further restricting to $M_{z} / t_{z}>-6$ and using $k_{q}=0$ and $k_{q}=-2 \pi / 3$ at the $\Gamma$ and $M$ points respectively for $q \in\{1,2,3\}$, we obtain $|u(\Gamma)\rangle=|\downarrow\rangle$, and

$$
|u(M)\rangle=\left\{\begin{array}{lll}
|\downarrow\rangle & \text { if } M_{z} / t_{z}>3 \\
|\uparrow\rangle & \text { if } M_{z} / t_{z}<3 .
\end{array}\right.
$$

Therefore, Eq. (15) dictates that the lower band has Chern number $\mathcal{C}=-2(\bmod 6)$ in the parameter range $-6<M_{z} / t_{z}<3$ (except $M_{z} / t_{z}=2$ at which the gap closes), and 0 otherwise, which we confirm numerically. Figure 2 shows the variation of the spin polarization of the lower energy band in the BZ. In the topological phase, the spin polarization at the corners of the BZ including at the $M$ point, is the negative of the polarization at the center, $\Gamma$.

One can now construct other Hamiltonians that host $|\mathcal{C}|=2,3$ phases by following a similar strategy. A nearest-neighbor two-band Hamiltonian on a triangular Bravais lattice with $\mathcal{C}=2$ is obtained by leveraging the Chern-spin polarization relation (15) for the $m=1$ symmetry. The symmetry condition places the exact same 
constraints on the $h_{z}$ coefficient as in Eq. (17), while the constraints on the $\sigma_{x}$ and $\sigma_{y}$ coefficients lead to

$$
\begin{aligned}
& h_{x}=2 t_{\text {so }} \sum_{q} \sin \left(k_{q}\right) \cos (2 q \pi / 3) ; \\
& h_{y}=2 t_{\text {so }} \sum_{q} \sin \left(k_{q}\right) \sin (2 q \pi / 3) .
\end{aligned}
$$

For small values of $|\mathbf{k}|$, it can be verified that

$$
h_{x} \sigma_{x}+h_{y} \sigma_{y} \propto\left[S_{z}(-\pi / 6) \mathbf{k}\right] \times \vec{\sigma} \cdot \hat{z}
$$

which is the Rashba SO coupling. The Chern number can again be evaluated directly by using the Chern-spin polarization relation. For $t_{\mathrm{so}} \neq 0$ and $M_{z}, t_{z}>0$, the spin at the $\Gamma$ point is $\left\langle\sigma_{z}\right\rangle_{\Gamma}=-1$. For $2<M_{z} / t_{z}<3$, the spin polarizations at the $M$ and the $X$ points are $\left\langle\sigma_{z}\right\rangle_{M}=-\left\langle\sigma_{z}\right\rangle_{X}=1$, which leads to a $\mathcal{C}=2$ phase. Note that this Hamiltonian exhibits a $|\mathcal{C}|=2$ phase for a smaller parameter range than was the case above when $m=2$. This could be attributed to the fact that in contrast to the $m=2$ symmetric Hamiltonian, the $m=1$ symmetric Rashba SO term does not enforce the Chern number to be even in general, and therefore a finer tuning of parameters is required.

The construction of tight-binding Hamiltonians with $|\mathcal{C}|=3$ follows a similar approach. Equation (15) for $m=$ 1 with the additional condition $\left\langle\sigma_{z}\right\rangle_{\Gamma}=\left\langle\sigma_{z}\right\rangle_{M}$ reduces to

$$
\mathcal{C}=\frac{n}{2}\left[\left\langle\sigma_{z}\right\rangle_{\Gamma}-\left\langle\sigma_{z}\right\rangle_{X}\right](\bmod 2 n)
$$

The additional condition is satisfied for

$$
\begin{aligned}
h_{z}= & M_{z}+2 t_{z}\left[\cos \left(k_{1}-k_{2}\right)+\cos \left(k_{2}-k_{3}\right)\right. \\
& \left.+\cos \left(k_{3}-k_{1}\right)\right] .
\end{aligned}
$$

To satisfy the $m=1$ symmetry, the coefficients of $\sigma_{x}$ and $\sigma_{y}$ are taken to have the same form as in Eq. (22). In the parameter range $-6<M_{z} / t_{z}<2$ and $t_{\mathrm{so}} \neq 0, t_{z}>0$, one obtains $\left\langle\sigma_{z}\right\rangle_{\Gamma}=-\left\langle\sigma_{z}\right\rangle_{X}=-1$, and therefore this Hamiltonian displays a $\mathcal{C}=-3$ phase. Next consider the $m=3$ symmetry case. Choosing $h_{z}$ as in Eq. (17), any two-band nearest-neighbor Hamiltonian is necessarily gapless, as $h_{x}, h_{y} \propto \sum_{q} \sin k_{q}$. One way to construct $h_{x}$ and $h_{y}$ coefficients satisfying the symmetry is to add next-nearest-neighbor SO coupling. One such choice is

$$
\begin{aligned}
& h_{x}=t_{\mathrm{so}} \sum_{q} \sin k_{q} \\
& h_{y}=t_{\mathrm{so}}\left[\sin \left(k_{1}-k_{2}\right)+\sin \left(k_{2}-k_{3}\right)+\sin \left(k_{3}-k_{1}\right)\right] .
\end{aligned}
$$

For small values of $|\mathbf{k}|$, this SO coupling has a complicated cubic dependence on $k_{x}, k_{y}$. For any $t_{\text {so }} \neq 0$, the total Hamiltonian displays a $|\mathcal{C}|=3$ phase for the parameter range $-6<M_{z} / t_{z}<2$.

We now use our approach for the construction of a $\mathcal{C}=2$ Hamiltonian using NNN terms. For $m=2$, we only require $\left\langle\sigma_{z}\right\rangle_{\Gamma}=-\left\langle\sigma_{z}\right\rangle_{M}$ to ensure $|\mathcal{C}|=2$. This is achieved by isotropic spin-dependent hopping term

$$
h_{z}=M_{z}+2 t_{z} \sum_{q=1,2} \cos k_{q}, \quad k_{q}=\mathbf{k} \cdot \mathbf{a}_{q}^{\mathrm{sq}}
$$

for $\left|M_{z}\right|<4\left|t_{z}\right|$, where $\mathbf{a}_{1}^{\mathrm{sq}}, \mathbf{a}_{2}^{\mathrm{sq}}$ are the lattice vectors of a square Bravais lattice. To satisfy the $m=2$ symmetry using nearest neighbor terms, the only possibility is $h_{x}, h_{y} \propto \cos k_{1}-\cos k_{2}$, which would lead to a gapless Hamiltonian. A gapped Hamiltonian can be constructed by introducing a weak diagonal hopping,

$$
\begin{aligned}
& h_{x}=t_{\mathrm{so}}\left(\cos k_{1}-\cos k_{2}\right), \\
& h_{y}=t_{\mathrm{so}}\left[\cos \left(k_{1}+k_{2}\right)-\cos \left(k_{1}-k_{2}\right)\right],
\end{aligned}
$$

which then displays $|\mathcal{C}|=2$ phase. Note the similarity with the $|\mathcal{C}|=3$ Hamiltonian on a triangular lattice satisfying the $m=3$ symmetry constructed above.

Following our approach, one may also attempt the construction of a square lattice Hamiltonian obeying the $m=1$ symmetry with $\mathcal{C}=2$. Such a Hamiltonian however requires strong next-nearest-neighbor $\sigma_{z}$ terms, as otherwise $\left\langle\sigma_{z}\right\rangle_{\Gamma}=\left\langle\sigma_{z}\right\rangle_{M}$ and $\left\langle\sigma_{z}\right\rangle_{\Gamma}=-\left\langle\sigma_{z}\right\rangle_{X}$ cannot be satisfied simultaneously. A closely related Hamiltonian with $|\mathcal{C}|=2$ bands has been constructed in Ref. [19].

The Hamiltonians we construct above enter a higher Chern number topological phase for infinitesimal values of the SO coupling strength $t_{\mathrm{so}}$, which is advantageous for experimental realization. Most of these Hamiltonians host $|\mathcal{C}|=2$ or $|\mathcal{C}|=3$ phases for $M_{z}=0$, which could be important for discovering materials that show quantum anomalous Hall effect in the total absence of external magnetic field. The construction of other topological Hamiltonians by leveraging symmetries for the purpose of ultracold atomic simulation has also been investigated in Ref. [68].

\section{REALIZING AND DETECTING A $|\mathcal{C}|=2$ PHASE WITH ULTRACOLD ATOMS}

\section{A. Overview of the proposed experimental setup}

We now describe how a topological Hamiltonian on a triangular lattice, described by Eqs. (17) and (18), could be realized using ultracold ${ }^{87} \mathrm{Rb}$ atoms. The scheme we present here only requires isolation of, and control over, three hyperfine levels forming a $\Lambda$ configuration for the realization of the SO coupling via Raman coherence, and therefore can be extended to all bosonic and fermionic atoms used in ultracold atomic experiments.

By applying a red-detuned laser sheet that is tightly focused along the $z$ axis, the atoms are confined to the $x y$ plane (Fig. 3). The atoms are then loaded onto a spin-independent triangular optical lattice potential [62], formed by three red-detuned $z$-polarized (linear $\pi$-polarized) "lattice" laser beams originating from the 


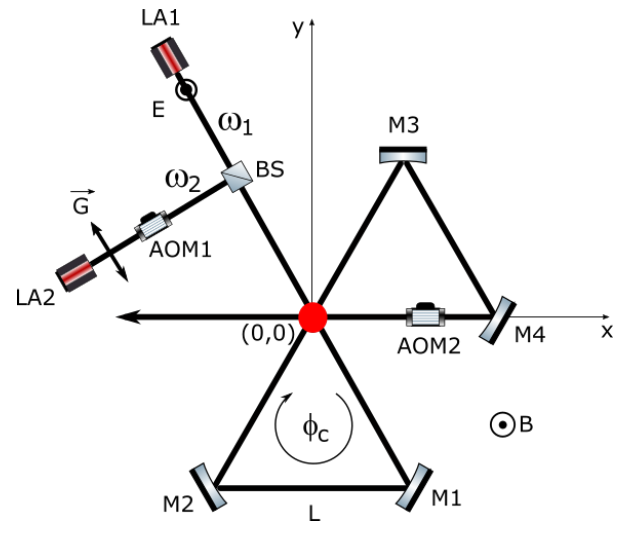

FIG. 3. Proposed experimental setup for the realization and the detection of a $|\mathcal{C}|=2$ topological phase. In the figure, LA1 and LA2 denote two lasers with the same frequency $\omega_{1}$ polarized out of plane (along $z$ axis) and in plane (in $x-y$ plane), respectively. BS denotes a 50 : 50 beam-splitter, M1-M4 are polarization-preserving mirrors, and AOM1 and AOM2 are acousto-optic modulators. An external magnetic field of strength $B$ is applied out of plane.

laser LA1 in Fig. 3. The scheme for inducing SO coupling, inspired by the experimental setup described in Ref. [54], makes use of two hyperfine levels of the groundstate manifold as the pseudospin- $1 / 2$ up $(|\uparrow\rangle)$ and down $(|\downarrow\rangle)$ states. To simulate SO coupling between these two states, a sufficiently strong external magnetic field needs to be applied in the $z$ direction, so that the hyperfine energy levels shift according to the quadratic Zeeman effect. Three "Raman" laser beams originating from the laser LA2 with linear in-plane polarization induce Raman transitions between the $|\uparrow\rangle$ and $|\downarrow\rangle$ levels via excited states. The Raman laser beams also generate an additional spin-dependent periodic potential.

For the detection of the Chern number, we design a scheme based on Bloch oscillations and TOF imaging that leverages the Chern-spin polarization relation. The spin polarization of the Bose-Einstein condensate of bosonic ultracold atoms can be obtained by releasing the atoms from the trap followed by a Stern-Gerlach measurement. The condensate can be moved from one point in the BZ to another by short Bloch oscillations. Such oscillations are induced by slowly accelerating the optical lattice, which is achieved by slowly and simultaneously chirping the frequencies of the lattice and Raman lasers. If the experiment is performed with fermionic atoms, then the Bloch oscillations are not required, as a direct spin-resolved TOF imaging suffices to obtain the spin polarization. Finally, the Chern number is calculated using the Chern-spin polarization relation.

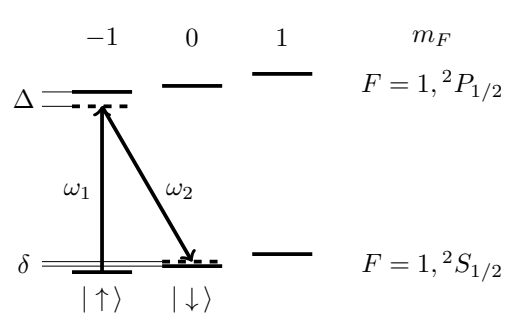

FIG. 4. The $\Lambda$-configuration, responsible for the Raman transition, formed by $|\uparrow\rangle$ and $|\downarrow\rangle$ states with the excited state $\left|F=1, m_{F}=-1\right\rangle$ in the $2 P_{1 / 2}$ multiplet. $F=2$ levels of the $2 P_{1 / 2}$ multiplet are not shown for simplicity.

\section{B. Derivation of the SO coupled Hamiltonian in the continuum}

\section{Laser configuration}

As per convention, the $z$-axis is the quantization axis of the atom, so that $J_{z}\left|J, m, F, m_{F}\right\rangle=\hbar(m+$ $\left.m_{F}\right)\left|J, m, F, m_{F}\right\rangle$, where $J_{z}=L_{z}+S_{z}+I_{z}$ is the $z$ component of the total angular momentum operator. The pseudospin-1/2 up and down states can then be chosen to correspond to two hyperfine levels of the ground state manifold, namely $|\uparrow\rangle:=\left|F=1, m_{F}=-1\right\rangle$ and $|\downarrow\rangle:=\left|F=1, m_{F}=0\right\rangle$, as depicted in Fig. 4 . The three lattice laser beams with amplitude $E_{0}$ propagate in the $x y$-plane with wavevectors

$$
\mathbf{k}_{1}=k(-1,0), \mathbf{k}_{2}=k\left(\frac{1}{2},-\frac{\sqrt{3}}{2}\right), \mathbf{k}_{3}=k\left(\frac{1}{2}, \frac{\sqrt{3}}{2}\right) .
$$

The frequency $\omega_{1}$ of the lattice laser beams is close to, but less than, the $D 1$ transition frequency. The three Raman laser beams are linear polarized in the $x y$ plane and propagate along the same directions as the lattice laser beams with amplitude $G_{0}$, and frequency $\omega_{2}=\omega_{1}+$ $\left(\epsilon_{\uparrow}-\epsilon_{\downarrow}\right)+\delta$, where $\epsilon_{\uparrow}-\epsilon_{\downarrow}$ is the Zeeman energy shift, and $\delta$ denotes the two-photon detuning. The frequency shift can be implemented with the help of an acousto-optic modulator AOM1, as shown in Fig. 3. The arm length $L$ needs to be adjusted such that the relative phase acquired by adjacent Raman laser beams is $\phi_{c}:=3 L \delta \omega / c=2 \pi / 3$.

The combination of the three in-plane polarized beams at any point in the $x y$-plane can be separated into superpositions of $\sigma_{+}$and $\sigma_{-}$circular polarization. In the spherical basis

$$
\mathbf{e}_{+}=\left(-\frac{1}{\sqrt{2}},-\frac{i}{\sqrt{2}}\right), \quad \mathbf{e}_{-}=\left(\frac{1}{\sqrt{2}},-\frac{i}{\sqrt{2}}\right),
$$

and using the definition $G_{ \pm}(\mathbf{r})=\mathbf{e}_{ \pm}^{*} \cdot \vec{G}(\mathbf{r})$, one obtains

$$
\mathbf{r} \cdot \vec{G}=-\frac{(x+i y)}{\sqrt{2}} G_{+}+\frac{(x-i y)}{\sqrt{2}} G_{-} .
$$

The $\sigma_{-}$-polarized component at frequency $\omega_{2}$ in combination with the linearly polarized field at frequency $\omega_{1}$ 
leads to Raman coherence in the $\Lambda$ system formed by $|\uparrow\rangle,|\downarrow\rangle$ and $|F,-1\rangle$ for each $F=1,2$ of the ${ }^{2} P_{1 / 2}$ multiplet (Fig. 4). All transitions to the level $\left|F=1, m_{F}=1\right\rangle$ in the ${ }^{2} S_{1 / 2}$ multiplet are suppressed due to a large twophoton detuning as a result of the quadratic Zeeman shift, and therefore can be ignored. The detuning $\Delta$ from the center of the D1 transition is large compared to both the hyperfine splitting and Zeeman energy shift in the ${ }^{2} P_{1 / 2}$ levels.

We now derive the symmetry properties of the electric fields, which will be used later to derive the optical lattice potential and the form of SO coupling induced by this laser configuration. The hyperfine splitting is much smaller than the $D 1$ energy gap, so that $\omega_{2}-\omega_{1} \ll \omega_{1}$; therefore, $\left|\mathbf{k}_{2}\right| \approx\left|\mathbf{k}_{1}\right|=k$ is a good assumption. The $E(\mathbf{r}, t)$ is the complex-valued electric field in the $z$ direction, and $\vec{G}(\mathbf{r}, t):=\left(G_{x}(\mathbf{r}, t), G_{y}(\mathbf{r}, t)\right)$ is the in-plane electric field along the $x$ and $y$ directions. One can now write $E(\mathbf{r}, t)=E(\mathbf{r}) e^{-i \omega_{1} t}, \vec{G}(\mathbf{r}, t)=\vec{G}(\mathbf{r}) e^{-i \omega_{2} t}$, where

$E(\mathbf{r})=E_{0} \sum_{j=1}^{3} e^{i \mathbf{k}_{j} \cdot \mathbf{r}}, \quad \vec{G}(\mathbf{r})=G_{0} \sum_{j=1}^{3} e^{i\left(\mathbf{k}_{j} \cdot \mathbf{r}+j \phi_{c}\right)} \hat{z} \times \mathbf{k}_{j}$.

Let

$$
\mathbf{a}_{1}=\frac{4 \pi}{3 k}(1,0), \quad \mathbf{a}_{2}=\frac{4 \pi}{3 k}\left(-\frac{1}{2}, \frac{\sqrt{3}}{2}\right) .
$$

The electric fields satisfy

$$
\begin{aligned}
E\left(\mathbf{r}+\mathbf{a}_{q}\right) & =e^{i 2 \pi / 3} E(\mathbf{r}) ; \\
G_{ \pm}\left(\mathbf{r}+\mathbf{a}_{q}\right) & =e^{i 2 \pi / 3} G_{ \pm}(\mathbf{r}), \quad q=1,2,3 .
\end{aligned}
$$

Equation (34) follows directly from the observation $\mathbf{k}_{j}$. $\mathbf{a}_{q}=2 \pi / 3 \bmod 2 \pi$ for all $q, j \in\{1,2,3\}$. Therefore, $\mathbf{a}_{1}, \mathbf{a}_{2}$ are the lattice vectors of the triangular optical lattice formed by the lattice lasers [62]. We also define $\mathbf{a}_{3}=-\mathbf{a}_{1}-\mathbf{a}_{2}$ as before. The reciprocal lattice vectors are

$$
\mathbf{b}_{1}=\sqrt{3} k\left(\frac{\sqrt{3}}{2}, \frac{1}{2}\right), \quad \mathbf{b}_{2}=\sqrt{3} k(0,1) .
$$

For the rotation by $2 \pi / 3$ around the $z$-axis implemented by

$$
\begin{aligned}
R^{2} & =R_{z}(2 \pi / 3)=\left[\begin{array}{cc}
\cos (2 \pi / 3) & -\sin (2 \pi / 3) \\
\sin (2 \pi / 3) & \cos (2 \pi / 3)
\end{array}\right] \\
& =\left[\begin{array}{cc}
-1 / 2 & -\sqrt{3} / 2 \\
\sqrt{3} / 2 & -1 / 2
\end{array}\right]
\end{aligned}
$$

one obtains

$$
E\left(R^{2} \mathbf{r}\right)=E(\mathbf{r}), \quad G_{ \pm}\left(R^{2} \mathbf{r}\right)=e^{i\left(\phi_{c} \mp 2 \pi / 3\right)} G_{ \pm}(\mathbf{r}) .
$$

Equation (37) follows from the rotational symmetry of the in-plane and out-of-plane field components. The outof-plane field is invariant under rotation by $2 \pi / 3$, which leads to the first equality in Eq. (37). The second equality can be derived as follows: First note that by symmetry of the experimental setup, the field $\vec{G}$ satisfies

$$
\vec{G}\left(R^{2} \mathbf{r}\right)=e^{i \phi_{c}} R^{2} \vec{G}(\mathbf{r}) .
$$

Using $\mathbf{e}_{ \pm}^{*} R^{2}=e^{\mp i 2 \pi / 3} \mathbf{e}_{ \pm}^{*}$, one obtains

$$
\begin{aligned}
G_{ \pm}\left(R^{2} \mathbf{r}\right) & =\mathbf{e}_{ \pm}^{*} \cdot \vec{G}\left(R^{2} \mathbf{r}\right) \\
& =e^{i \phi_{c}} \mathbf{e}_{ \pm}^{*} \cdot R^{2} \vec{G}(\mathbf{r}) \\
& =e^{i\left(\phi_{c} \mp 2 \pi / 3\right)} \mathbf{e}_{ \pm}^{*} \vec{G}(\mathbf{r}) \\
& =e^{i\left(\phi_{c} \mp 2 \pi / 3\right)} G_{ \pm}(\mathbf{r}),
\end{aligned}
$$

as desired.

\section{Optical lattice potential}

We now compute the optical lattice potential due to the lattice laser beams. The frequency $\omega_{1}$ can be chosen to be close to $D 1$ transition frequency so as to ensure that D2 transitions can be safely ignored. This assumption is made only for simplicity; the scheme works even if both D1 and D2 transitions have comparable contributions. The Rabi frequency of oscillation due to the optical lattice lasers is

$$
\begin{aligned}
& \hbar \Omega_{\uparrow, 0, F}(\mathbf{r})=\langle F,-1|e z| \uparrow\rangle E(\mathbf{r}), \\
& \hbar \Omega_{\downarrow, 0, F}(\mathbf{r})=\langle F, 0|e z| \downarrow\rangle E(\mathbf{r}),
\end{aligned}
$$

where $F$ corresponds to the excited level in the ${ }^{2} P_{1 / 2}$ space. The optical lattice potential generated by the laser with $\omega_{1}$ frequency alone is

$$
V(\mathbf{r})=\sum_{F=1,2} \frac{\hbar}{4 \Delta}\left|\Omega_{\sigma, 0, F}(\mathbf{r})\right|^{2}
$$

where $\Delta$ is the detuning. Note that since $\Omega_{\sigma, 0, F} \propto E(\mathbf{r})$, then $V(\mathbf{r}) \propto|E(\mathbf{r})|^{2}$. The lattice potential is generated by $\pi$-polarized light and therefore is spin-independent (see discussion above Eq. (44) in Ref. [69] for a rigorous justification). The details of the exact potential can be found in Ref. [62]. The contribution to the continuum Hamiltonian due to lattice lasers alone is

$$
H_{\text {lat }}=-\frac{\hbar^{2} \nabla^{2}}{2 m}+V(\mathbf{r}) \text {. }
$$

\section{Spin-dependent potential}

In contrast to the out-of-plane polarized lattice lasers, the Raman lasers add a spin-dependent component to the optical lattice potential. The dipole potential for alkali atoms in the state $\left(F, m_{F}\right)$ due to $D 1$ transitions is given by the formula [70]

$$
V_{m_{F}}(\mathbf{r})=\frac{\pi c^{2} \Gamma}{2 \omega_{0}^{3}}\left(\frac{1-\mathcal{P} g_{F} m_{F}}{\Delta}\right) I(\mathbf{r}),
$$


where the detuning $\Delta$ is with respect to the center of the $D 1$ line, $g_{F}$ is the Landé factor, $m_{F}$ is the relevant magnetic spin state of the atom, and $\mathcal{P}= \pm 1$ and $\mathcal{P}=0$ represent the local polarization $\sigma^{ \pm}$and $\pi$, respectively, of the light field relative to the chosen quantization axis. For the $|\downarrow\rangle$ state, $m_{F}=0$, and therefore the second term vanishes. However, for the $|\uparrow\rangle$, we have $m_{F}=-1$ and therefore the second term is non zero. We define

$$
W(\mathbf{r})=V_{\uparrow}(\mathbf{r})-V_{\downarrow}(\mathbf{r})=\frac{\pi c^{2} \Gamma g_{F}}{2 \omega_{0}^{3} \Delta} \sum_{i=\sigma_{ \pm}} \mathcal{P}_{i} I_{i}(\mathbf{r})
$$

as additional spin-dependent dipole potential due to Raman lasers. This contributes the term $W(\mathbf{r}) \sigma_{z} / 2$ to the total Hamiltonian. The $V_{\uparrow}(\mathbf{r})+V_{\downarrow}(\mathbf{r})$ term can be ignored because it is spin independent and can be absorbed in $V(\mathbf{r})$.

\section{Spin-orbit coupling}

The Raman laser beams, together with the lattice laser beams, induce Raman coupling between the $|0\rangle$ and $|1\rangle$ states. Both $F=1$ and $F=2$ levels in the ${ }^{2} P_{1 / 2}$ space contribute to Raman resonance. The effective twophoton Rabi frequency is

$$
\Omega_{\mathrm{R}}(\mathbf{r})=\frac{1}{2 \Delta} \sum_{F=1,2} \Omega_{\downarrow,-, F}^{*}(\mathbf{r}) \Omega_{\uparrow, 0, F}(\mathbf{r}),
$$

where

$$
\hbar \Omega_{\downarrow,-, F}(\mathbf{r})=\frac{1}{\sqrt{2}}\langle F,-1|e(x-i y)| \downarrow\rangle G_{-}(\mathbf{r}) .
$$

Note that the two-photon Rabi frequency satisfies

$$
\Omega_{\mathrm{R}}(\mathbf{r}) \propto E(\mathbf{r}) G_{-}^{*}(\mathbf{r}) .
$$

It follows from Eqs. (34) and (37) that

$$
\begin{aligned}
\Omega_{\mathrm{R}}\left(\mathbf{r}+\mathbf{a}_{q}\right) & =\Omega_{\mathrm{R}}(\mathbf{r}), \quad q=1,2,3, \\
\Omega_{\mathrm{R}}\left(R^{2} \mathbf{r}\right) & =e^{-i\left(\phi_{c}+2 \pi / 3\right)} \Omega_{\mathrm{R}}(\mathbf{r}) .
\end{aligned}
$$

Then, the contribution to the Hamiltonian due to Raman resonance is [71]

$V_{\mathrm{R}}(\mathbf{r})=-\hbar\left[\begin{array}{cc}\frac{\delta}{2}+\sum_{F} \frac{\left|\Omega_{\downarrow, 0, F}(\mathbf{r})\right|^{2}}{4 \Delta} & \frac{\Omega_{\mathrm{R}}^{*}(\mathbf{r})}{2} \\ \frac{\Omega_{\mathrm{R}}(\mathbf{r})}{2} & -\frac{\delta}{2}+\sum_{F} \frac{\left|\Omega_{\uparrow,-, F}(\mathbf{r})\right|^{2}}{4 \Delta}\end{array}\right]$,

where $\delta$ is the two-photon detuning. Note that the terms

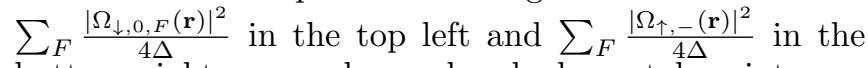
bottom right corner have already been taken into account when calculating the optical lattice potential and the spin-dependent correction to it.

After combining contributions from all factors, the total effective Hamiltonian in the continuum becomes

$$
H=H_{\text {lat }}+\left(\frac{W(\mathbf{r})}{2}-\frac{\hbar \delta}{2}\right) \sigma_{z}-\left(\frac{\hbar \Omega_{\mathrm{R}}(\mathbf{r})}{2} \sigma^{+}+\text {H.c. }\right) \text {, }
$$

where $H_{\text {lat }}$ is the sum of the kinetic energy and the lattice potential generated by the lattice lasers, and the coefficients $W(\mathbf{r})$ and $\Omega_{\mathrm{R}}(\mathbf{r})$ depend on experimentally adjustable parameters, namely the amplitudes $E_{0}, G_{0}$, and the single-photon and two-photon detunings $\Delta$ and $\delta$ respectively.

\section{Derivation of the tight-binding Hamiltonian}

The effective tight-binding Hamiltonian is constructed by restricting the continuum Hamiltonian to the Wannier states of the lowest energy band. We show through a detailed analysis of the symmetry properties of these Wannier states that the resulting Bloch Hamiltonian has matrix coefficients of the form in Eqs. (17) and (18). The key step involves taking advantage of the symmetry properties of the Wannier functions as well as the electric field configuration to obtain the phases acquired by atoms while hopping in various directions accompanied by (possible) spin flips.

Consider the Bloch eigenstates $\psi_{n, \mathbf{k}}$ of the lattice Hamiltonian $H_{\text {lat }}$ corresponding to $n$th band and crystal momentum k. Next, construct the Wannier states

$$
\phi_{n, j_{1}, j_{2}}^{\sigma}(\mathbf{r})=\frac{1}{\sqrt{N}} \sum e^{-i \mathbf{k} \cdot \mathbf{r}} \psi_{n, \mathbf{k}}^{\sigma}\left(j_{1} \mathbf{a}_{1}+j_{2} \mathbf{a}_{2}\right),
$$

defined for all pairs of integers $\vec{j}:=\left(j_{1}, j_{2}\right)$. From here onwards, we will restrict attention to the Wannier functions of the lowest energy band $n=0$, and assume that the Wannier functions $\phi_{j_{1}, j_{2}}^{\sigma}(\mathbf{r}):=\phi_{0, j_{1}, j_{2}}^{\sigma}(\mathbf{r})$ are symmetric under six-fold rotation generated by $R$, that is

$$
\phi_{\overrightarrow{0}}^{\sigma}(\mathbf{r})=\phi_{\overrightarrow{0}}^{\sigma}(R \mathbf{r}) .
$$

Because the lattice Hamiltonian $H_{\text {lat }}$ is spin-independent, one also has

$$
\phi_{j_{1}, j_{2}}^{\uparrow}(\mathbf{r})=\phi_{j_{1}, j_{2}}^{\downarrow}(\mathbf{r})
$$

Further, since the lattice potential $V(\mathbf{r})$ is real, it is possible to use a gauge in which $\psi_{n,-\mathbf{k}}(\mathbf{r})=\psi_{n, \mathbf{k}}^{*}(\mathbf{r})$, which leads to the conclusion that the Wannier functions $\phi^{\sigma}$ are real-valued. Note that the Wannier functions have been constructed using the band eigenstates of $H_{\text {lat }}$, which is a topologically trivial Hamiltonian. Therefore, as long as a continuous gauge for the eigenstates $\psi_{n, \mathbf{k}}$ is chosen, the Wannier functions are expected to be exponentially localized [72].

The lattice Hamiltonian term $H_{\text {lat }}$ leads to isotropic hopping without spin-flip with strength $t_{\mathrm{hop}}$,

$$
\widehat{H}_{\text {hop }}=-t_{\text {hop }} \sum_{\left\langle\vec{j}, \vec{j}^{\prime}\right\rangle} \hat{\Phi}_{\vec{j}}^{\dagger} \hat{\Phi}_{\vec{j}^{\prime}}
$$

where $\left\langle\vec{j}, \vec{j}^{\prime}\right\rangle$ denote the restriction that $\vec{j}, \vec{j}^{\prime}$ are nearestneighbor sites on the triangular lattice, $\hat{\Phi}_{\vec{j}}^{\dagger}=\left[\begin{array}{cc}\hat{\phi}_{\vec{j}, \uparrow}^{\dagger} & \hat{\phi}_{\vec{j}, \downarrow}^{\dagger}\end{array}\right]$ 
are arrays of creation operators corresponding to the Wannier orbitals, and

$$
-t_{\mathrm{hop}}=\int d^{2} \mathbf{r}\left(\phi_{\overrightarrow{0}}^{\downarrow}\right)^{*}(\mathbf{r}) H_{\mathrm{lat}}(\mathbf{r}) \phi_{\vec{a}_{1}}^{\uparrow}(\mathbf{r})
$$

where $\vec{a}_{1}:=(1,0), \vec{a}_{2}:=(0,1)$, and $\vec{a}_{3}:=(-1,-1)=$ $-\vec{a}_{1}-\vec{a}_{2}$ are the integer coordinates of lattice vectors $\mathbf{a}_{1}, \mathbf{a}_{2}$, and $\mathbf{a}_{3}$ in the units of $\mathbf{a}_{1}$ and $\mathbf{a}_{2}$, and $\overrightarrow{0}:=(0,0)$. Note that the Wannier functions are real-valued, and so Eq. (52) would still hold if $\left(\phi_{0,0}^{\downarrow}\right)^{*}$ was replaced by $\phi_{0,0}^{\downarrow}$ in the integrand.

The optical lattice formed by a $z$-polarized laser has a spin-independent correction due to the in-plane polarized lasers. We claim without proof that the correction to the hopping strength due to this contribution is also isotropic. Whether this claim is true or false does not affect further analysis, because the Wannier basis of the optical lattice potential is governed by $z$-polarized lasers, and the spin-independent correction only adds a factor proportional to the identity to the Hamiltonian in momentum space that does not affect the eigenvectors or their topology.

Next consider the contribution to the tight-binding Hamiltonian due to the term $W(\mathbf{r}) \sigma_{z} / 2$ in the continuum Hamiltonian. Once again, the term leads to an equal $\sigma_{z^{-}}$ type hopping between all nearest neighbors,

$$
\widehat{H}_{\mathrm{zhop}, 1}=t_{z} \sum_{\left\langle\vec{j}, \vec{j}^{\prime}\right\rangle} \hat{\Phi}_{\vec{j}}^{\dagger} \sigma_{z} \hat{\Phi}_{\vec{j}^{\prime}}
$$

where

$$
t_{z}:=\frac{1}{2} \int d^{2} \mathbf{r}\left(\phi_{\overrightarrow{0}}^{\downarrow}\right)^{*}(\mathbf{r}) W(\mathbf{r}) \phi_{\vec{a}_{1}}^{\uparrow}(\mathbf{r})
$$

Apart from a $\sigma_{z}$-hopping term, $W(\mathbf{r}) \sigma_{z} / 2$ also contributes an on-site $\sigma_{z}$ mass term, given by

$$
\widehat{H}_{\mathrm{zhop}, 2}=m_{z} \sum_{\vec{j}} \hat{\Phi}_{\vec{j}}^{\dagger} \sigma_{z} \hat{\Phi}_{\vec{j}}
$$

where

$$
m_{z}:=\frac{1}{2} \int d^{2} \mathbf{r}\left(\phi_{\overrightarrow{0}}^{\downarrow}\right)^{*}(\mathbf{r}) W(\mathbf{r}) \phi_{\overrightarrow{0}}^{\uparrow}(\mathbf{r})
$$

The Raman process is responsible for inducing hopping with spin-flip in the tight-binding Hamiltonian. The amplitude of the hopping term between the Wannier centers $(0,0)$ and $\left(j_{1}, j_{2}\right)$ can be computed using the formula

$$
t_{\mathrm{so}}\left(j_{1}, j_{2}\right)=\frac{\hbar}{2} \int d^{2} \mathbf{r}\left(\phi_{\overrightarrow{0}}^{\downarrow}\right)^{*}(\mathbf{r}) \Omega_{\mathrm{R}}(\mathbf{r}) \phi_{j_{1}, j_{2}}^{\uparrow}(\mathbf{r}) .
$$

Because $\phi^{\uparrow}=\phi^{\downarrow}$ and $\phi^{\sigma}$ is real-valued, one obtains

$$
\begin{aligned}
t_{\mathrm{so}}\left(-\vec{a}_{q}\right) & =\frac{\hbar}{2} \int d^{2} \mathbf{r}\left(\phi_{\overrightarrow{0}}^{\downarrow}\right)^{*}(\mathbf{r}) \Omega_{\mathrm{R}}(\mathbf{r}) \phi_{-\vec{a}_{q}}^{\uparrow}(\mathbf{r}) \\
& =\frac{\hbar}{2} \int d^{2} \mathbf{r}\left(\phi_{\overrightarrow{0}}^{\downarrow}\right)(\mathbf{r}) \Omega_{\mathrm{R}}(\mathbf{r})\left(\phi_{-\vec{a}_{q}}^{\uparrow}\right)^{*}(\mathbf{r}) \\
& =\frac{\hbar}{2} \int d^{2} \mathbf{r}\left(\phi_{-\vec{a}_{q}}^{\uparrow}\right)^{*}(\mathbf{r}) \Omega_{\mathrm{R}}(\mathbf{r}) \phi_{\overrightarrow{0}}^{\uparrow}(\mathbf{r}) \\
& =\frac{\hbar}{2} \int d^{2} \mathbf{r}\left(\phi_{-\vec{a}_{q}}^{\downarrow}\right)^{*}(\mathbf{r}) \Omega_{\mathrm{R}}(\mathbf{r}) \phi_{\overrightarrow{0}}^{\uparrow}(\mathbf{r}) \\
& =\frac{\hbar}{2} \int d^{2} \mathbf{r}\left(\phi_{\overrightarrow{0}}^{\downarrow}\right)^{*}(\mathbf{r}) \Omega_{\mathrm{R}}(\mathbf{r}) \phi_{\vec{a}_{q}}^{\uparrow}(\mathbf{r}) \\
& =t_{\mathrm{so}}\left(\vec{a}_{q}\right) .
\end{aligned}
$$

Using $\mathbf{a}_{3}=R^{2} \mathbf{a}_{2}=R^{4} \mathbf{a}_{1}$ along with the translation invariance of the Wannier functions $\phi_{j_{1}, j_{2}}(\mathbf{r})=\phi_{\overrightarrow{0}}(\mathbf{r}-$ $\left.j_{1} \mathbf{a}_{1}-j_{2} \mathbf{a}_{2}\right)$, one obtains

$$
\begin{aligned}
\phi_{\vec{a}_{2}}^{\sigma}\left(R^{2} \mathbf{r}\right) & =\phi_{\overrightarrow{0}}^{\sigma}\left(R^{2} \mathbf{r}-\mathbf{a}_{2}\right)=\phi_{\overrightarrow{0}}^{\sigma}\left(R^{2}\left(\mathbf{r}-\mathbf{a}_{1}\right)\right)=\phi_{\overrightarrow{0}}^{\sigma}\left(\mathbf{r}-\mathbf{a}_{1}\right) \\
& =\phi_{\vec{a}_{1}}^{\sigma}(\mathbf{r})
\end{aligned}
$$

and

$$
\begin{aligned}
\phi_{\vec{a}_{3}}^{\sigma}\left(R^{2} \mathbf{r}\right) & =\phi_{\overrightarrow{0}}^{\sigma}\left(R^{2} \mathbf{r}-\mathbf{a}_{3}\right)=\phi_{\overrightarrow{0}}^{\sigma}\left(R^{2}\left(\mathbf{r}-\mathbf{a}_{2}\right)\right)=\phi_{\overrightarrow{0}}^{\sigma}\left(\mathbf{r}-\mathbf{a}_{2}\right) \\
& =\phi_{\vec{a}_{2}}^{\sigma}(\mathbf{r}) .
\end{aligned}
$$

The hopping amplitudes $t_{\mathrm{so}}\left(\vec{a}_{2}\right)$ and $t_{\mathrm{so}}\left(\vec{a}_{3}\right)$ can now be calculated as follows:

$$
\begin{aligned}
t_{\mathrm{so}}\left(\vec{a}_{2}\right) & =\frac{\hbar}{2} \int d^{2} \mathbf{r}\left(\phi_{\overrightarrow{0}}^{\downarrow}\right)^{*}(\mathbf{r}) \Omega_{\mathrm{R}}(\mathbf{r}) \phi_{\vec{a}_{2}}^{\uparrow}(\mathbf{r}) \\
& =\frac{\hbar}{2} \int d^{2} \mathbf{s}\left(\phi_{\overrightarrow{0}}^{\downarrow}\right)^{*}\left(R^{2} \mathbf{s}\right) \Omega_{\mathrm{R}}\left(R^{2} \mathbf{s}\right) \phi_{\vec{a}_{2}}^{\uparrow}\left(R^{2} \mathbf{s}\right) \\
& =\frac{\hbar}{2} \int d^{2} \mathbf{s}\left(\phi_{\overrightarrow{0}}^{\downarrow}\right)^{*}(\mathbf{s}) e^{-i\left(\phi_{c}+2 \pi / 3\right)} \Omega_{\mathrm{R}}(\mathbf{s}) \phi_{\vec{a}_{1}}^{\uparrow}(\mathbf{s}) \\
& =e^{-i\left(\phi_{c}+2 \pi / 3\right)} t_{\mathrm{so}}\left(\vec{a}_{1}\right),
\end{aligned}
$$

where the change of variable $\mathbf{r}=R^{2} \mathbf{s}$ is employed in the first step and Eqs. (46) and (59) are used in the later steps. Note that since $R$ is a rotation, the Jacobian of the transformation is 1 . A similar calculation yields

$$
t_{\mathrm{so}}\left(\vec{a}_{3}\right)=e^{-i\left(\phi_{c}+2 \pi / 3\right)} t_{\mathrm{so}}\left(\vec{a}_{2}\right)
$$

The on-site spin-flipping term does not survive for $\phi_{c}=$ $2 \pi / 3$. This follows from the relation

$$
\begin{aligned}
t_{\mathrm{so}}(\overrightarrow{0}) & =\frac{\hbar}{2} \int d^{2} \mathbf{r}\left(\phi_{\overrightarrow{0}}^{\downarrow}\right)^{*}(\mathbf{r}) \Omega_{\mathrm{R}}(\mathbf{r}) \phi_{\overrightarrow{0}}^{\uparrow}(\mathbf{r}) \\
& =\frac{\hbar}{2} \int d^{2} \mathbf{s}\left(\phi_{\overrightarrow{0}}^{\downarrow}\right)^{*}\left(R^{2} \mathbf{s}\right) \Omega_{\mathrm{R}}\left(R^{2} \mathbf{s}\right) \phi_{\overrightarrow{0}}^{\uparrow}\left(R^{2} \mathbf{s}\right) \\
& =\frac{\hbar}{2} \int d^{2} \mathbf{s}\left(\phi_{\overrightarrow{0}}^{\downarrow}\right)^{*}(\mathbf{s}) e^{-i\left(\phi_{c}+2 \pi / 3\right)} \Omega_{\mathrm{R}}(\mathbf{s}) \phi_{\overrightarrow{0}}^{\uparrow}(\mathbf{s}) \\
& =e^{-i\left(\phi_{c}+2 \pi / 3\right)} t_{\mathrm{so}}(\overrightarrow{0})
\end{aligned}
$$

which implies that $t_{\mathrm{so}}(\overrightarrow{0})=0$ for $\phi_{c}=2 \pi / 3$.

One can redefine $\phi^{\downarrow}$ by a global gauge transformation such that $t_{\text {so }}\left(\vec{a}_{3}\right)$ is real and positive. From here onwards, 
we set $t_{\mathrm{so}}:=t_{\mathrm{so}}\left(\vec{a}_{3}\right)$. Now the total contribution to the tight-binding Hamiltonian due to the Raman process is

$$
\begin{aligned}
\widehat{H}_{\text {so }}=\sum_{\vec{j}} \sum_{q=1}^{3} t_{\text {so }} & \left\{\hat { \Phi } _ { \vec { j } } ^ { \dagger } \left[\cos (-2 q \pi / 3) \sigma_{x}\right.\right. \\
& \left.\left.+\sin (-2 q \pi / 3) \sigma_{y}\right] \hat{\Phi}_{\vec{j}+\vec{a}_{q}}+\text { H.c. }\right\} .
\end{aligned}
$$

Finally, the two-photon detuning leads to on-site $\sigma_{z}$ term with strength $-\hbar \delta \sigma_{z} / 2$ independent of the value of the phase difference $\phi_{c}$,

$$
\widehat{H}_{\mathrm{det}}=-\frac{\hbar \delta}{2} \sum_{\vec{j}} \hat{\Phi}_{\vec{j}}^{\dagger} \sigma_{z} \hat{\Phi}_{\vec{j}},
$$

It is safe to assume that the two-photon detuning can be adjusted to be small enough so that any resulting nn hopping can be ignored.

Combining all terms, one obtains the full tight-binding Hamiltonian Eqs. (51), (53), (55), (69), and (70). Because the Hamiltonian is number conserving, one may write the single-particle Hamiltonian as

$$
H=H_{\text {hop }}+H_{\text {zhop }}+H_{\text {so }}+H_{\text {det }},
$$

where

$$
\begin{aligned}
H_{\mathrm{hop}}= & -t \sum_{\vec{j}, q}\left(|\vec{j}\rangle\left\langle\vec{j}+\vec{a}_{q}\right|+\text { H.c. }\right), \\
H_{\text {zhop }}= & t_{z} \sum_{\vec{j}, q}\left(|\vec{j}\rangle\left\langle\vec{j}+\vec{a}_{q}\right| \sigma_{z}+\text { H.c. }\right), \\
H_{\mathrm{so}}= & t_{\mathrm{so}} \sum_{\vec{j}, q}\left\{| \vec { j } \rangle \langle \vec { j } + \vec { a } _ { q } | \left[\cos (-2 q \pi / 3) \sigma_{x}\right.\right. \\
& \left.\left.+\sin (-2 q \pi / 3) \sigma_{y}\right]+ \text { H.c. }\right\}, \\
H_{\mathrm{det}}= & \left(m_{z}-\frac{\hbar \delta}{2}\right) \sum_{\vec{j}}|\vec{j}\rangle\langle\vec{j}| \sigma_{z}
\end{aligned}
$$

To obtain the tight-binding Hamiltonian in momentum space, define

$$
V_{q}=\sum_{\vec{j}}|\vec{j}\rangle\left\langle\vec{j}+\vec{a}_{q}\right|, \quad q=1,2,3,
$$

and the momentum states

$$
|\mathbf{k}\rangle=\frac{1}{\sqrt{N}} \sum_{\vec{j}} e^{i \mathbf{k} \cdot \mathbf{j}}|\vec{j}\rangle
$$

where again $\vec{j}=\left(j_{1}, j_{2}\right)$ and $\mathbf{j}=j_{1} \mathbf{a}_{1}+j_{2} \mathbf{a}_{2}$. It is easy to verify that

$$
V_{q}|\mathbf{k}\rangle=e^{i k_{q}}|\mathbf{k}\rangle, \quad V_{q}^{\dagger}|\mathbf{k}\rangle=e^{-i k_{q}}|\mathbf{k}\rangle .
$$

After omitting the term proportional to identity in spin space, and $M_{z}:=m_{z}-\frac{\hbar \delta}{2}$, one obtains the SO Hamiltonian in momentum space $H(\mathbf{k})=\mathbf{H}(\mathbf{k}) \cdot \boldsymbol{\sigma}=h_{x} \sigma_{x}+$ $h_{y} \sigma_{y}+h_{z} \sigma_{z}$, with the coefficients $h_{x}, h_{y}, h_{z}$ as in Eqs. (17) and (18). This is the second main result of the present work.

We conclude the description of the experimental scheme for the realization of SO coupling and the desired topological state on a triangular lattice by a qualitative comparison to the scheme used in Ref. [54]. Despite the apparent similarity, our scheme differs substantially from the scheme used in Ref. [54] for the square lattice. The lattice potential in our scheme is generated by $\pi$ polarized lasers, which are truly spin-independent. More important, the Raman potential in our scheme has the same periodicity as the optical lattice in the standard gauge. In contrast, the scheme in Ref. [54] leads to a Raman potential that has twice the periodicity of the optical lattice before a particular gauge transformation is implemented. Such a gauge transformation is not possible in a triangular lattice due to the lack of sublattice symmetry. We turn to the detection of Chern number in the next section.

\section{Chern number via Zeeman Spectroscopy and Bloch oscillations}

We now show how the Chern-spin polarization relation, (15), can be leveraged to obtain the Chern number $(\bmod 6)$ of the lower band wavefunction. The triangular SO-coupled lattice requires $n=3$ and $m=2$, and the relation can be further simplified to $\mathcal{C}=2\left(\left\langle\sigma_{z}\right\rangle_{M}-\right.$ $\left.\left\langle\sigma_{z}\right\rangle_{\Gamma}\right)(\bmod 6)$. Typically, the spin-independent hopping term $-2 t_{\text {hop }}\left[\cos \left(k_{1}\right)+\cos \left(k_{2}\right)+\cos \left(k_{3}\right)\right]$ of the Hamiltonian, which was omitted in Eq. (20), dominates as far as the energy eigenvalues are concerned. Therefore the ${ }^{87} \mathrm{Rb}$ atoms condense to form a Bose-Einstein condensate (BEC) at the $\Gamma$ point in the center of the BZ that minimizes this term, as shown in Fig. 1. The ratio of the populations in the $|\uparrow\rangle,|\downarrow\rangle$ levels obtained by SternGerlach imaging (i.e. Zeeman spectroscopy) can be used to infer the spin polarization at the $\Gamma$ point.

The spin polarization at the point $M$ in the BZ can be obtained by first performing a short Bloch oscillation to move the BEC adiabatically [63] from the $\Gamma$ point to the $M$ point. For a triangular lattice, the coordinates of the $M$ point, shown in Fig. 1, are given by

$$
\begin{aligned}
M & =\left(-\mathbf{b}_{1}+2 \mathbf{b}_{2}\right) / 3=\sqrt{3} k\left[-\frac{1}{3}(0,1)+\frac{2}{3}\left(\frac{\sqrt{3}}{2}, \frac{1}{2}\right)\right] \\
& =(k, 0) .
\end{aligned}
$$

To map the condensate from the $\Gamma$ point to the $M$ point, it suffices to accelerate the lattice along the $x$ direction with some magnitude $a$, which is accomplished by varying the frequency of the laser beams travelling in the $-x$ direction. Recall that when all three $\omega_{1}$ beams meet at the origin $(0,0)$ in phase, one of the lattice sites coincides with the origin, taken to be the center of the lattice. How does the center of the lattice shift when the third beam 
(propagating along $-x$ ) reaches the origin with a phase difference $\varphi$ added by AOM2 with respect to the initial configuration? Due to the symmetry of the system, the center must shift to a point $\mathbf{r}_{c}=(r, 0)$ along the $x$-axis, satisfying

$$
\varphi+\mathbf{k}_{1} \cdot \mathbf{r}_{c}=\mathbf{k}_{2} \cdot \mathbf{r}_{c}=\mathbf{k}_{3} \cdot \mathbf{r}_{c}
$$

The second equality is automatically satisfied for any point $\mathbf{r}_{c}$ along the $x$-axis. Solving the first equality leads to

$$
\varphi+k(-1,0) \cdot(r, 0)=k\left(\frac{1}{2},-\frac{\sqrt{3}}{2}\right) \cdot(r, 0),
$$

which gives $r=2 \varphi / 3 k$. To achieve acceleration $a$ along the $x$-direction, one needs

$$
\varphi(t)=\frac{3 k}{2}\left(\frac{1}{2} a t^{2}\right)=\frac{3 k a t^{2}}{4} .
$$

Assuming that the frequency variation is applied at $t=0$, the condition can be translated to

$$
\int_{t=0}^{t} \Delta \omega(t) d t=\varphi(t)
$$

which on differentiating yields the frequency difference as a function of the time required to achieve the phase difference $\varphi(t)$ at the origin,

$$
\Delta \omega(t)=\left(\frac{3 k a}{2}\right) t
$$

Therefore, to achieve acceleration $\alpha$ along $+x$ direction, the frequency of the third beam needs to be changed at a linear rate, with $d \Delta \omega / d t=3 k a / 2$.

The time required to move the BEC from the $\Gamma$ point to the $M$ point can be calculated as follows. The rate of change of crystal momentum is given by

$$
\hbar \frac{d \mathbf{k}}{d t}=-m a
$$

so that to reach $(k, 0)$ from $(0,0)$, the time required is

$$
T=\frac{\hbar k}{m a}
$$

where $m$ is the mass of the atom. The acceleration $a$ can be chosen to be arbitrary, but the adiabaticity condition [73] must be satisfied,

$$
T \gg \frac{\hbar\|d H / d t\|}{\Delta E^{2}} .
$$

Typically, we expect the SO-coupling strength $t_{\text {so }} \ll$ $t_{\text {hop }}$, so that the energy gap is determined by $t_{\text {so }}$ and the bandwidth by $t_{\text {hop }}$. The condition on $a$ then becomes

$$
\frac{\hbar k}{m a} \gg \frac{\hbar t_{\mathrm{hop}}}{t_{\mathrm{so}}^{2}} \Longrightarrow a \ll \frac{k t_{\mathrm{so}}^{2}}{m t_{\mathrm{hop}}} .
$$

If the Hamiltonian in Eq. (20) is realized using fermionic atoms, and assuming that the density of atoms is adjusted so that the band is half-filled, then the Chernspin polarization relation can still be leveraged to measure the Chern number experimentally. In contrast to bosons, the fermions at half-filling occupy the entire lower energy band due to Fermi-Dirac statistics. The spin polarization at both the $\Gamma$ and the $M$ points can then be directly obtained by standard TOF Stern-Gerlach imaging, which involves first turning off the lattice and $\mathrm{Ra}$ man lasers to let the atoms evolve freely for a time $t$ in an external magnetic field, and then imaging separately the population of each hyperfine level. At the end of the time interval, the atoms with crystal momentum $\mathbf{k}$ reach approximately the point $\mathbf{k} t / m \hbar$ in real space, so that the spin amplitude at $\mathbf{k}$ can be obtained from the population difference at the point $\mathbf{k} t / m \hbar$ in real space. In this case, the spatial profile of the population difference between the two levels will resemble the pattern in Fig. 2.

\section{CONCLUSIONS}

In this work, we have shown that the origin of some $|\mathcal{C}|=2,3$ phases can be traced to combined real spacespin rotation symmetries for SO-coupled lattice gases. This insight leads to a Chern-spin polarization relation (15) that allows for the determination of the Chern number by measuring the particle spin polarization at only a small number of points in the BZ. In the simple setting of nearest-neighbor hopping, we demonstrated that triangular Bravais lattices can accommodate higher Chern numbers compared to their square counterparts. We leveraged this result to provide a detailed proposal for the experimental realization of a $|\mathcal{C}|=2$ phase on a triangular lattice using Raman-induced SO coupling in ultracold atomic gases. The trivial and topological phases can then be distinguished using TOF Zeeman imaging for fermions and a combination of Bloch oscillations and TOF Zeeman imaging for bosons. Our scheme for the detection of the Chern number suggests that Bloch oscillations and Zeeman spectroscopy could be adapted to a large class of SO-coupled systems for the detection of Chern insulators in ultracold atoms.

The Chern-spin polarization relation and the tightbinding models that we constructed illustrate that symmetries with larger values of $m$ induce favorable conditions for the realization of higher Chern number states, which should galvanize the search for such states in systems with unconventional SO coupling. In the present analysis, the values of $|\mathcal{C}|$ are restricted to 2,3 due to the smaller cardinality of the symmetry groups that divide the BZ in 4 or 6 equal parts (c.f. Fig. 1).

To achieve yet higher values of the Chern number, it would be natural to explore systems with larger symmetry groups, such as those supported on non-Bravais lattices (i.e. Bravais lattices with attached basis) [74], systems with anti-unitary symmetries [35, 75], and be- 
yond. We hope to pursue these investigations in future work. It remains to be seen how our methods can be extended to multiband systems, including time-reversal invariant systems which have vanishing Chern number and systems with intrinsic topological order. Although Zak-Chern relations may be trivial for such systems, the topology can often be inferred from the wave function at highly symmetric crystal momenta [75].

\section{ACKNOWLEDGMENTS}

This research was supported by the Natural Sciences and Engineering Research Council of Canada and the Alberta Major Innovation Fund. A. A. acknowledges support through a Killam 2020 Postdoctoral Fellowship.
[1] X.-L. Qi and S.-C. Zhang, Rev. Mod. Phys. 83, 1057 (2011).

[2] S. Ryu, A. P. Schnyder, A. Furusaki, and A. W. W. Ludwig, New J. Phys. 12, 065010 (2010).

[3] Y.-F. Wang, H. Yao, C.-D. Gong, and D. N. Sheng, Phys. Rev. B 86, 201101(R) (2012).

[4] M. Barkeshli and X.-L. Qi, Phys. Rev. X 2, 031013 (2012).

[5] A. M. Cook, C. Hickey, and A. Paramekanti, Phys. Rev. B 90, 085145 (2014).

[6] J. Song, Y.-Y. Zhang, Y. Li, and Q.-F. Sun, J. Phys. Condens. Matter 27, 045601 (2015).

[7] X. Zhang and S.-C. Zhang, in Micro- and Nanotechnology Sensors, Systems, and Applications IV, edited by T. George, M. S. Islam, and A. Dutta, International Society for Optics and Photonics (SPIE, 2012), vol. 8373.

[8] L. Li, X. Kong, X. Chen, J. Li, B. Sanyal, and F. M. Peeters, Appl. Phys. Lett. 117, 143101 (2020).

[9] G. Chen, A. L. Sharpe, E. J. Fox, Y.-H. Zhang, S. Wang, L. Jiang, B. Lyu, H. Li, K. Watanabe, T. Taniguchi, et al., Nature 579, 56 (2020).

[10] J. Ge, Y. Liu, J. Li, H. Li, T. Luo, Y. Wu, Y. Xu, and J. Wang, Natl. Sci. Rev. 7, 1280 (2020).

[11] Y.-F. Zhao, R. Zhang, R. Mei, L.-J. Zhou, H. Yi, Y.Q. Zhang, J. Yu, R. Xiao, K. Wang, N. Samarth, et al., Nature 588, 419 (2020).

[12] S. A. Skirlo, L. Lu, Y. Igarashi, Q. Yan, J. Joannopoulos, and M. Soljačić, Phys. Rev. Lett. 115, 253901 (2015).

[13] M. Trescher and E. J. Bergholtz, Phys. Rev. B 86, 241111(R) (2012).

[14] B. Huang, C. F. Chan, and M. Gong, Phys. Rev. B 91, $134512(2015)$.

[15] H. Jiang, Z. Qiao, H. Liu, and Q. Niu, Phys. Rev. B 85, 045445 (2012).

[16] J. Wang, B. Lian, H. Zhang, Y. Xu, and S.-C. Zhang, Phys. Rev. Lett. 111, 136801 (2013).

[17] A. M. Cook and A. Paramekanti, Phys. Rev. Lett. 113, 077203 (2014).

[18] F. Wang and Y. Ran, Phys. Rev. B 84, 241103(R) (2011).

[19] D. Sticlet, F. Piéchon, J.-N. Fuchs, P. Kalugin, and P. Simon, Phys. Rev. B 85, 165456 (2012).

[20] Z. Liu, E. J. Bergholtz, H. Fan, and A. M. Läuchli, Phys. Rev. Lett. 109, 186805 (2012).

[21] S. Yang, Z.-C. Gu, K. Sun, and S. Das Sarma, Phys. Rev. B 86, 241112(R) (2012).

[22] S.-Y. Lee, J.-H. Park, G. Go, and J. H. Han, J. Phys. Soc. Japan 84, 064005 (2015).

[23] D. Peter, N. Y. Yao, N. Lang, S. D. Huber, M. D. Lukin, and H. P. Büchler, Phys. Rev. A 91, 053617 (2015).

[24] R.-J. Slager, A. Mesaros, V. Juričić, and J. Zaanen, Na- ture Physics 9, 98 (2013).

[25] D. Sticlet and F. Piéchon, Phys. Rev. B 87, 115402 (2013).

[26] C. H. Lee, M. Claassen, and R. Thomale, Phys. Rev. B 96, 165150 (2017).

[27] A. M. Cook, Phys. Rev. B 94, 205135 (2016).

[28] Z. Zhang, J.-Y. You, X.-Y. Ma, B. Gu, and G. Su, arXiv preprint arXiv:2010.07670 (2020).

[29] R. O. Umucalılar, H. Zhai, and M. O. Oktel, Phys. Rev. Lett. 100, 070402 (2008).

[30] L. B. Shao, S.-L. Zhu, L. Sheng, D. Y. Xing, and Z. D. Wang, Phys. Rev. Lett. 101, 246810 (2008).

[31] F. Li, L. B. Shao, L. Sheng, and D. Y. Xing, Phys. Rev. A 78, 053617 (2008).

[32] C. Zhang, Phys. Rev. A 82, 021607(R) (2010).

[33] E. Zhao, N. Bray-Ali, C. J. Williams, I. B. Spielman, and I. I. Satija, Phys. Rev. A 84, 063629 (2011).

[34] E. Alba, X. Fernandez-Gonzalvo, J. Mur-Petit, J. K. Pachos, and J. J. Garcia-Ripoll, Phys. Rev. Lett. 107, 235301 (2011)

[35] X.-J. Liu, K. T. Law, T. K. Ng, and P. A. Lee, Phys. Rev. Lett. 111, 120402 (2013).

[36] D. A. Abanin, T. Kitagawa, I. Bloch, and E. Demler, Phys. Rev. Lett. 110, 165304 (2013).

[37] S.-L. Zhu, Z.-D. Wang, Y.-H. Chan, and L.-M. Duan, Phys. Rev. Lett. 110, 075303 (2013).

[38] A. Dauphin and N. Goldman, Phys. Rev. Lett. 111, 135302 (2013).

[39] L. Wang, A. A. Soluyanov, and M. Troyer, Phys. Rev. Lett. 110, 166802 (2013).

[40] P. Hauke, M. Lewenstein, and A. Eckardt, Phys. Rev. Lett. 113, 045303 (2014).

[41] F. Grusdt, D. Abanin, and E. Demler, Phys. Rev. A 89, 043621 (2014).

[42] S.-T. Wang, D.-L. Deng, and L.-M. Duan, Phys. Rev. Lett. 113, 033002 (2014).

[43] D.-L. Deng, S.-T. Wang, and L.-M. Duan, Phys. Rev. A 90, 041601(R) (2014).

[44] H. M. Price, O. Zilberberg, T. Ozawa, I. Carusotto, and N. Goldman, Phys. Rev. B 93, 245113 (2016).

[45] W.-W. Zhang, B. C. Sanders, S. Apers, S. K. Goyal, and D. L. Feder, Phys. Rev. Lett. 119, 197401 (2017).

[46] B.-Z. Wang, Y.-H. Lu, W. Sun, S. Chen, Y. Deng, and X.-J. Liu, Phys. Rev. A 97, 011605(R) (2018).

[47] M. Atala, M. Aidelsburger, J. T. Barreiro, D. Abanin, T. Kitagawa, E. Demler, and I. Bloch, Nat. Phys. 9, 795 (2013).

[48] G. Jotzu, M. Messer, R. Desbuquois, M. Lebrat, T. Uehlinger, D. Greif, and T. Esslinger, Nature 515, 237 (2014). 
[49] L. Duca, T. Li, M. Reitter, I. Bloch, M. Schleier-Smith, and U. Schneider, Science 347, 288 (2015).

[50] M. Aidelsburger, M. Lohse, C. Schweizer, M. Atala, J. T. Barreiro, S. Nascimbène, N. R. Cooper, I. Bloch, and N. Goldman, Nat. Phys. 11, 162 (2015).

[51] T. Li, L. Duca, M. Reitter, F. Grusdt, E. Demler, M. Endres, M. Schleier-Smith, I. Bloch, and U. Schneider, Science 352, 1094 (2016).

[52] N. Fläschner, B. S. Rem, M. Tarnowski, D. Vogel, D.S. Lühmann, K. Sengstock, and C. Weitenberg, Science 352, 1091 (2016).

[53] S. Nakajima, T. Tomita, S. Taie, T. Ichinose, H. Ozawa, L. Wang, M. Troyer, and Y. Takahashi, Nat. Phys. 12, 296 (2016).

[54] Z. Wu, L. Zhang, W. Sun, X.-T. Xu, B.-Z. Wang, S.-C. Ji, Y. Deng, S. Chen, X.-J. Liu, and J.-W. Pan, Science 354, 83 (2016).

[55] W. Sun, B.-Z. Wang, X.-T. Xu, C.-R. Yi, L. Zhang, Z. Wu, Y. Deng, X.-J. Liu, S. Chen, and J.-W. Pan, Phys. Rev. Lett. 121, 150401 (2018).

[56] G.-B. Jo, J. Guzman, C. K. Thomas, P. Hosur, A. Vishwanath, and D. M. Stamper-Kurn, Phys. Rev. Lett. 108, 045305 (2012).

[57] C. K. Thomas, T. H. Barter, T.-H. Leung, S. Daiss, and D. M. Stamper-Kurn, Phys. Rev. A 93, 063613 (2016).

[58] C. Gross and I. Bloch, Science 357, 995 (2017).

[59] P. Soltan-Panahi, D.-S. Lühmann, J. Struck, P. Windpassinger, and K. Sengstock, Nature Physics 8, 71 (2012).

[60] X. Li and W. V. Liu, Reports on Progress in Physics 79, 116401 (2016).

[61] Y.-J. Lin, K. Jiménez-García, and I. B. Spielman, Nature
471, 83 (2011).

[62] C. Becker, P. Soltan-Panahi, J. Kronjäger, S. Dörscher, K. Bongs, and K. Sengstock, New J. Phys. 12, 065025 (2010).

[63] M. Ben Dahan, E. Peik, J. Reichel, Y. Castin, and C. Salomon, Phys. Rev. Lett. 76, 4508 (1996).

[64] O. Morsch, J. H. Müller, M. Cristiani, D. Ciampini, and E. Arimondo, Phys. Rev. Lett. 87, 140402 (2001).

[65] M. Cristiani, O. Morsch, J. H. Müller, D. Ciampini, and E. Arimondo, Phys. Rev. A 65, 063612 (2002).

[66] D. McKay, M. White, and B. DeMarco, Phys. Rev. A 79, 063605 (2009).

[67] C. Fang, M. J. Gilbert, and B. A. Bernevig, Phys. Rev. B 86, 115112 (2012).

[68] Y. Kuno, I. Ichinose, and Y. Takahashi, Scientific Reports 8, 10699 (2018).

[69] D. A. Steck, Rubidium 87 d line data (2001), Accessed: 2020-09-18.

[70] R. Grimm, M. Weidemüller, and Y. B. Ovchinnikov, in Advances in atomic, molecular, and optical physics (Elsevier, 2000), vol. 42, pp. 95-170.

[71] E. Brion, L. H. Pedersen, and K. Mølmer, J. Phys. A Math. Theor. 40, 1033 (2007).

[72] D. Monaco, G. Panati, A. Pisante, and S. Teufel, Communications in Mathematical Physics 359, 61 (2018).

[73] A. Messiah, Quantum mechanics, vol. II (North-Holland, Amsterdam, 1962).

[74] J. Höller and A. Alexandradinata, Phys. Rev. B 98, 024310 (2018).

[75] L. Fu and C. L. Kane, Phys. Rev. B 76, 045302 (2007). 